\title{
A SIMPLE MODEL OF AC HOPPING CONDUCTIVITY IN DISORDERED SOLIDS
}

\author{
Jeppe C. DYRE \\ Institute of Mathematics and Physics, IMFUFA, University of Roskilde, \\ Postbox 260, DK-4000 Roskilde, Denmark
}

Received 13 November 1984; revised manuscript received 16 January 1985; accepted for publication 19 February 1985

\begin{abstract}
Using the CTRW approximation the simplest possible nontrivial model of ac hopping conductivity in disordered solids, is constructed. The model predicts universality of the frequency-dependent conductivity, independent of temperature, chemical composition, and conductivity mechanism, in reasonably good agreement with experiments. It is shown that all qualitative features of experimental ac hopping conductivity can be understood within the model.
\end{abstract}

Hopping conductivity in disordered solids has been studied for several years. The basic characteristic of ac hopping conductivity is a power-law frequency dependence of the real part of the conductivity, $\sigma(\omega)$, at high frequencies, $\omega$,

$\operatorname{Re} \sigma(\omega) \propto \omega^{s}$,

as was first observed by Pollak and Geballe in 1961 [1]. They found that conductivity in n-type doped crystalline silicon is well described by an exponent $s$ equal to 0.8 . Since then, power-law frequency-dependent conductivity has been observed in a wide variety of disordered solids, including amorphous semiconductors, organic solids, and oxide glasses [2]. One finds values of $s$ between 0.5 and 1.0, often close to one [3]. AC hopping conductivity has been reviewed by several authors [3-6].

When discussing hopping conductivity one usually thinks of electronic or polaronic hopping. For instance, conductivity in amorphous semiconductors is believed to be due to phonon-assisted quantum-mechanical tunneling of electrons/polarons between localized states in the mobility gap [3]. But hopping conductivity arises also in the entirely different context of ionic conductivity in oxide glasses (reviewed in refs. $[5,7,8]$ ). Glass ionic conductivity also obeys eq. (1). Actually, there seems to be a close relationship between ionic and electronic conductivity in oxide glasses. Both give rise to exactly identical dielectric loss peaks, as has been emphasized by Isard and by Owen $[9,5]$. This fact is very surprising because ionic conductivity is a classical, thermally activated process, while electronic hopping conductivity involves quantum-mechanical tunneling. This "paradox" presents a challenge to any theory of hopping conductivity in disordered solids.

An approach which is frequently adopted in the calculation of ac loss in amorphous semiconductors, is the pair approximation. It is assumed that ac loss is mainly due to electron jumps between pairs of localized states [10]. In this model there is an approximate power-law behaviour of the conductivity at high frequencies with the exponent $s$ given by [11]

$s \equiv \mathrm{d} \ln \operatorname{Re} \sigma(\omega) / \mathrm{d} \ln \omega=1+4 / \ln \left(\omega \tau_{\mathrm{ph}}\right)$.

Here $\tau_{\text {ph }}$ is a characteristic phonon time (or order $10^{-12} \mathrm{~s}$ ). For usual laboratory frequencies eq. (2) yields a value of $s$ close to 0.8 , which in the early days of amorphous physics was thought to be the universal exponent of ac hopping conductivity [11]. However, the pair approximation suffers from several weaknesses. First or all, the approximation certainly does not apply at low frequencies, and the transition to dc conductivity cannot be understood within this approximation. But even at high frequencies there are problems. Eq. (2) predicts that $s$ is a slowly decreasing function of the frequency. This has never been observed, if $s$ varies at all it is an increasing function 
of the frequency [5]. Also, in order to explain the frequently observed $s$ values close to one (e.g. in most amorphous solids at low temperatures) one has to assume values of $\tau_{\mathrm{ph}}$ smaller than $10^{-20} \mathrm{~s}$. But this is totally unrealistic.

A sounder approach to hopping conductivity is to consider the actual random walk of the charge carriers in the disordered solid. This approach was pioneered by Scher and Lax in their important papers of 1973 [12]. They proposed to model the disordered solid as a regular lattice with randomly varying jump frequencies $\gamma$. The frequency-dependent conductivity was then calculated by means of the continuous time random walk approximation (CTRW). Klafter and Silbey have later shown that the CTRW is formally rigorous [13]. The approximation in ref. [12] enters via the Scher-Lax method to construct the so-called hopping-time distribution function. Today, the Scher-Lax approach is known to be equivalent to the Hartree approximation, the simplest possible nontrivial mean-field approximation [14]. There exist more accurate (and more involved) mean-field approximations (e.g. EMA [14]), but we shall here use the Hartree approximation in which the conductivity is given by $[14,15]$

$\sigma(\omega)=K\left[-\mathrm{i} \omega+\left\langle(\gamma+\mathrm{i} \omega)^{-1}\right\rangle^{-1}\right]$.

In eq. (3) $K$ is a constant (depending on charge carrier concentration, average jump distance, temperature, etc), and \langle\rangle denotes the average over the jump frequency distribution $p(\gamma)$.

By means of eq. (3) $\sigma(\omega)$ is determined solely by $p(\gamma)$. We now address the problem of deriving the simplest possible $p(\gamma)$ which still contains the essential physics of hopping conductivity. In the case of electronic tunneling between localized states, the jump frequency is essentially given by [16]

$\gamma \propto r^{3 / 2} \mathrm{e}^{-2 \alpha r} \mathrm{e}^{-w / k T}$,

where $r$ is the jump distance, $\alpha$ is the decay parameter for the wavefunctions of the localized states, $W$ is the energy difference between the two states, $k$ is the Boltzmann constant, and $T$ is the temperature. If $W$ varies according to a Boltzmann distribution $[p(W) \propto$ $\left.\exp \left(-W / k T_{0}\right)\right]$ one arrives at $p(\gamma) \propto \gamma^{-\delta}$ with a nontrivial $\delta$ [this, of course, gives a non-trivial $s$ in eq. (1)] [17]. Here we shall assume ad hoc that $W$ varies completely randomly. If furthermore the jump distance probability is given by a geometrical $r^{2}$-factor, it is not hard to see from eq. (4) that $p(\gamma)$ is given by $\gamma^{-1}$ times some logarithmic terms. If jumps to more than one nearest neighbour are allowed, the resulting jump frequency distribution is the above distribution convoluted with itself a number of times. Again one ends up with a $\gamma^{-1}$-term times some logarithmic terms which are not very important. Thus, a good approximation to the correct jump frequency distribution is

$p(\gamma)=(1 / \ln \lambda)(1 / \gamma), \quad \gamma_{\min }<\gamma<\gamma_{\max }$,

where two cut-off's, $\gamma_{\min }$ and $\gamma_{\max }$, have been introduced, and $\lambda=\gamma_{\max } / \gamma_{\min }$. It is now easy to understand the surprising similarity between electronic and ionic hopping conductivity: A randomly varying ion jump activation energy will produce exactly the same jump frequency distribution as in the electronic case [eq. (5)], and thereby the same $\sigma(\omega)$. This is an important conclusion of this letter.

Substituting eq. (5) into eq. (3) we find

$\sigma(\omega)=K\left\{-i \omega+i \omega \ln \lambda\left[\ln \left(\frac{1+i \omega / \gamma_{\min }}{1+i \omega / \gamma_{\max }}\right)\right]^{-1}\right\}$

Eq. (6) implies that the conductivity rises from the de conductivity, $\sigma_{0}$, to a high frequency conductivity, $\sigma_{\infty}$, in the frequency-range between $\gamma_{\min }$ and $\gamma_{\max }$. While $\gamma_{\min }$ is seen experimentally as defining the transition from dc to ac conductivity, there is in most cases no sign of any leveling off of the conductivity at high frequencies. It is therefore desirable to eliminate the artificial cut-off at $\gamma_{\max }$. But just letting $\gamma_{\max }$ go to infinity does not work since $\sigma(\omega)$ diverges in this limit. Instead we use the following renormalization procedure: The dc conductivity is [from eq. (6)] given by

$\sigma_{0}=K \ln \lambda /\left(\gamma_{\min }^{-1}-\gamma_{\max }^{-1}\right)$.

For large $\lambda$ the first term in eq. (6) can be ignored, so substituting $K \ln \lambda$ from eq. (7) into eq. (6) we find

$$
\begin{gathered}
\sigma(\omega)=\sigma_{0} i \omega\left(\gamma_{\min }^{-1}-\gamma_{\max }^{-1}\right) \\
\times\left[\ln \left(\frac{1+i \omega / \gamma_{\min }}{1+i \omega / \gamma_{\max }}\right)\right]^{-1} .
\end{gathered}
$$

Now it is possible to let $\gamma_{\max }$ go to infinity. In this limit we finally find 


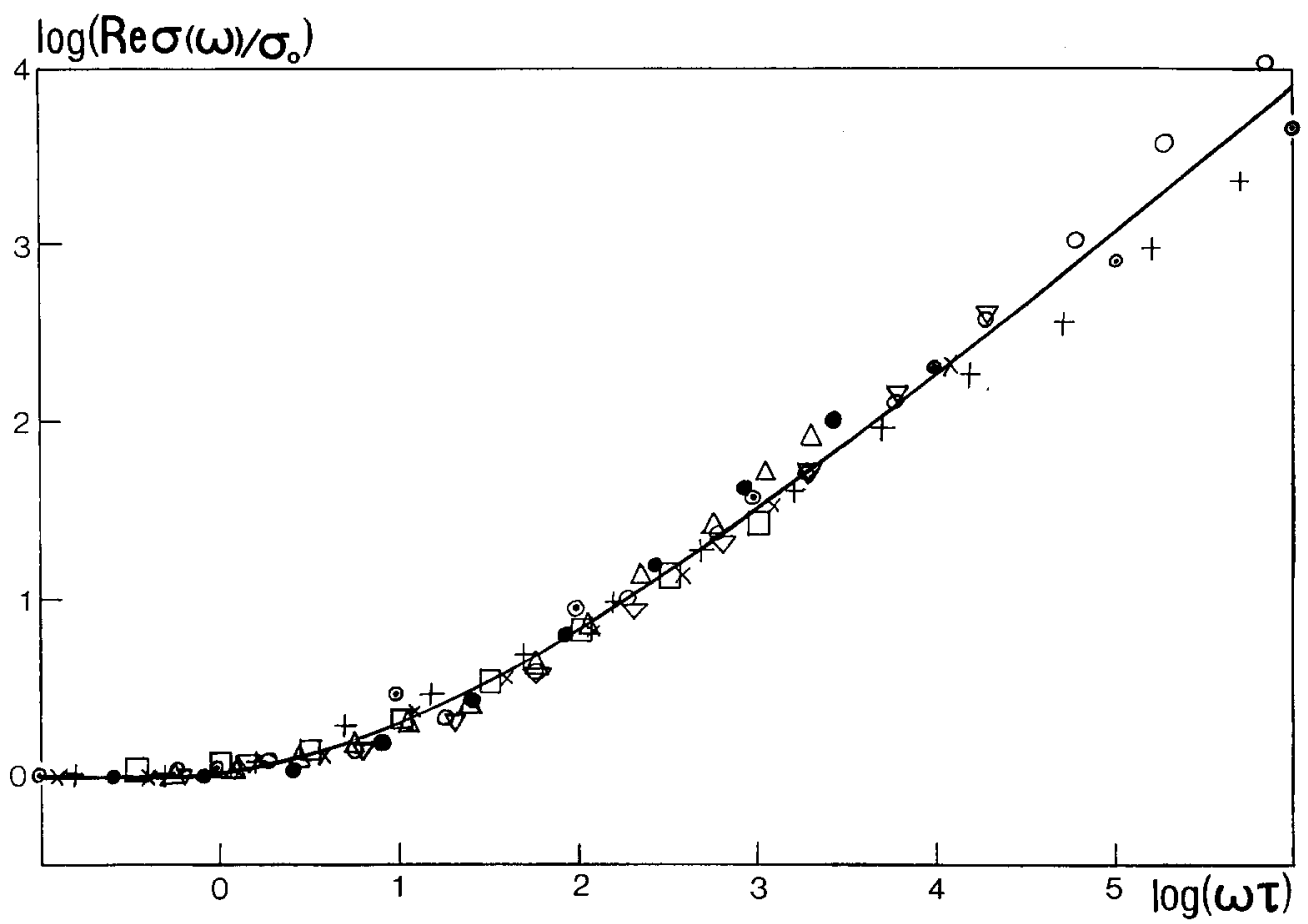

Fig. 1. Predictions of the model (full curve) and some experimental data for various hopping systems. The phenomenological time $\tau$ has been chosen for each data set to fit the curve as well as possible ( $\tau$ varies between $10^{-7} \mathrm{~s}$ and $10^{3} \mathrm{~s}$ ). The data represent hopping conductivity in: (1) n-doped crystalline silicon (X) [1] (using the universal conductivity curve [12]), (2) sputtered films of arsenic at $295 \mathrm{~K}(\bullet)$ [3], (3) ionically conducting glasses (e.g. sodium silicates etc.) $(\odot)$ ( $\sigma_{0}$ has been calculated using Namikawa's formula [19]),[5], (4) glow discharge silicon at $283 \mathrm{~K}(\Delta)$ [20], (5) silicon monoxide at $241 \mathrm{~K}(+)$ [21], (6) amorphous germanium at 88.5 $\mathrm{K}$ () [22], (7) $\mathrm{Mn}_{1.8} \mathrm{Ni}_{0.6} \mathrm{Co}_{0.6} \mathrm{O}_{4}$ at various temperatures (using the universal curve of fig. 8a of ref. [23]) ( $\nabla$ ), (8) monolayer of stearic acid at $300 \mathrm{~K}(0)[24]$.

$\sigma(\omega)=\sigma_{0} i \omega \tau[\ln (1+\mathrm{i} \omega \tau)]^{-1}$,

where $\tau=\gamma_{\min }^{-1}$. The real part of the conductivity is given by

$\operatorname{Re} \sigma(\omega)=\sigma_{0} \frac{\omega \tau \arctan (\omega \tau)}{\left\{\ln \left[1+(\omega \tau)^{2}\right]^{1 / 2}\right\}^{2}+[\arctan (\omega \tau)]^{2}}$

The model predicts a universal frequency dependence of the conductivity (except for scale transformations), independent of chemical composition and temperature. Universality of $\sigma(\omega)$ in suitably reduced units has been frequently discussed in connection with hopping conductivity $[5,9,12,18]$, but always in more restricted context. In fig. 1 the predicted real part of the conductivity is drawn together with data (randomly selected from the literature) for various hopping systems. A careful inspection of fig. 1 reveals that the ambitious claim of complete universality is not in agreement with experiment. But the model certainly reproduces the overall trend of the data. At high frequencies the conductivity follows an approximate power law. The exponent $s$ is from eq. (10) given by

$s \approx 1-2 / \ln (\omega \tau)$.

Exact values of $s$ for different $\omega \tau$ are given in table 1 . As in the pair approximation $s$ is always smaller than one. Contrary to the pair approximation, but in agreement with experiment [5], the theory predicts that $s$ is a slightly increasing function of the frequency. At very high frequencies $s$ approaches one. Thus, as a consequence of the universality the theory predicts that whenever an exponent $s$ close to one is observed, the dc conductivity is very small compared to the measured ac conductivity. 
Table 1

Model predictions of the exponent $s$ defined by $s=d \ln \operatorname{Re} \sigma(\omega) /$ $\mathrm{d} \ln \omega$ at various $\omega \tau$. Also, the approximate expression of $s$, $1-2 / \ln (\omega \tau)$, is included [eq. (11)].

\begin{tabular}{lll}
\hline$\omega \tau$ & $s$ & $1-2 / \ln (\omega \tau)$ \\
\hline $10^{2}$ & 0.62 & 0.57 \\
$10^{4}$ & 0.79 & 0.78 \\
$10^{6}$ & 0.86 & 0.86 \\
$10^{8}$ & 0.89 & 0.89 \\
$10^{10}$ & 0.91 & 0.91 \\
$10^{12}$ & 0.93 & 0.93 \\
$10^{14}$ & 0.94 & 0.94 \\
$10^{16}$ & 0.95 & 0.95 \\
\hline
\end{tabular}

Eq. (7) predicts proportionality between $\sigma_{0}$ and $\gamma_{\min }=\tau^{-1}$ (for $\gamma_{\min } \ll \gamma_{\max }$ ). This proportionality is due to the fact that the jump frequency distribution eq. (5) strongly emphasizes the smallest jump frequencies, which are also the most important for $\sigma_{0}$ because they partially act as traps. Proportionality between $\sigma_{0}$ and the dielectric loss peak frequency (which in the present model is of order $\tau^{-1}$ ) has been known experimentally for several years $[8,9,19]$. Actually, the constant $K$ is proportional to $T^{-1}$ [14], so for a given sample we have

$\sigma_{0}=(p / T) \tau^{-1}$,

where $p$ is a temperature-independent constant. Substituting eq. (12) into eq. (9) we get

$\sigma(\omega, T)=\mathrm{i} \omega p\left[T \ln \left(1+\frac{\mathrm{i} \omega p}{T \sigma_{0}(T)}\right)\right]^{-1}$.

In all hopping systems $\sigma_{0}$ is zero at zero temperature, so from eqs. (11) and (12) we conclude that the exponent $s$ (at a definite frequency) goes to one as the temperature goes to zero. This is what is always observed [3]. Eq. (13) prescribes how to displace the universal $\sigma(\omega)$-curve as the temperature varies [12]. In fig. 2 is shown the predicted and measured $\sigma(\omega)$ at some different temperatures for amorphous germanium. The agreement between theory and experiments is good.

In conclusion, a simple model of ac hopping conductivity has been constructed. The model suggests that the physics of ac hopping conductivity may be simpler than has hitherto been recognized. The model is semi-phenomenological in the sense that the absolute values of $\sigma_{0}$ and $\tau$ are not predicted. Three ap-

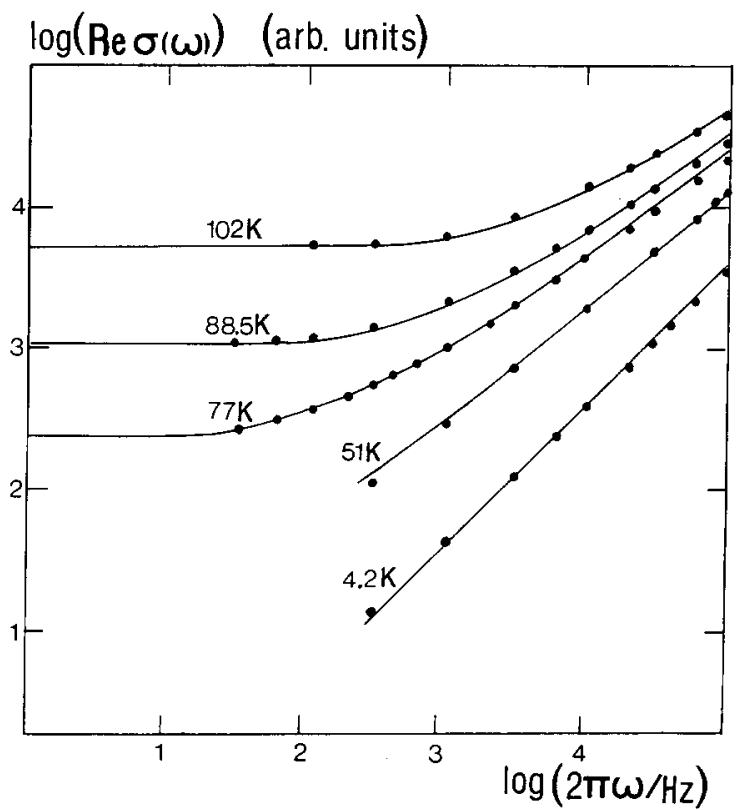

Fig. 2. Comparison between the prediction of eq. (13) (full curves) and measurements on amorphous germanium at various temperatures [22]. The universal conductivity curve has been fitted to the data at $77 \mathrm{~K}$, and then displaced according to eq. (13) to fit the data at the other temperatures.

proximations are involved in the model: (1) the CTRW approximation, (2) a jump frequency distribution proportional to $\gamma^{-1}$, and (3) the existence of a sharp cut-off at $\gamma_{\min }$. As regards the last point, it should be noted that in the case of nearest-neighbour hopping between localized states, a sharp cut-off is indeed realistic. This is a consequence of the exponential factor in the Hertz nearest-neighbour distance distribution: $p(r) \propto r^{2} \exp \left(-c r^{3}\right)$ [15]. At low temperatures, when variable-range hopping is believed to take place [11], the sharp cut-off becomes unrealistic. Also, in the case of glass ionic conductivity, a sharp jump frequency cut-off may well be questioned. However, the cut-off problem only affects the transition from de to ac conductivity.

The model predicts universality of the frequencydependent conductivity (in suitable units), independent of chemical composition and temperature. Although exact universality is not observed (fig. 1), the claim of universality has a number of interesting qualitative consequences. First of all, electronic, polaronic and ionic hopping conductivity in disordered solids should be similar. Any hopping system should have a 
power-law frequency dependence of the ac conductivity. The exponent $s$ is predicted to be a slighly increasing function of the frequency, and smaller than but close to one. Considering the temperature-dependence of $\sigma(\omega)$, the universality implies in particular temperature-independent dielectric loss peaks. Also, $s$ is predicted to be a decreasing function of temperature, which approaches one as $T$ goes to zero. In a $\log -\log$ plot like fig. 1 or fig. 2 , the ac conductivity is less temperature-dependent than the dc conductivity, and in the limit of $s=1$ the ac conductivity becomes practically temperature-independent. All of the above predictions are in agreement with experiments $[2-5,25]$. Thus, the proposed semi-phenomenological model correctly predicts the qualitative features of ac hopping conductivity.

I acknowledge helpful comments and suggestions from N.B. Olsen, T. Christensen and K. Snadeflink.

\section{References}

[1] M. Pollak and T.H. Geballe, Phys. Rev. 122 (1961) 1742.

[2] A.K. Jonscher, Nature 267 (1977) 673.

[3] A.R. Long, Adv. Phys. 31 (1982) 553.

[4] R.M. Hill and A.K. Jonscher, J. Non-Cryst. Solids 32 (1979) 53.

[5] A.E. Owen, J. Non-Cryst. Solids 25 (1977) 372.
[6] H. Böttger and V.V. Bryskin, Phys. Stat. Sol, 78b (1976) 9,415 ; 113b (1982) 9.

[7] K. Hughes and J.O. Isard, in: Physics of electrolytes, Vol. 1, ed.J.H. Hladik (Academic Press, New York, 1972) p. 351 .

[8] M. Tomozawa, in: Treatise of materials science, Vol. 12, ed. M. Tomozawa (Academic Press, New York, 1977) p. 283.

[9] J.O. Isard, J. Non-Cryst. Solids 4 (1970) 357.

[10] I.G. Austin and N.F. Mott, Adv. Phys. 18 (1969) 41.

[11] N.F. Mott and E.A. Davis, Electronic processes in noncrystalline materials (Clarendon Press, Oxford, 1971).

[12] H. Scher and M. Lax, Phys. Rev. B7 (1973) 4491, 4502.

[13] J. Klafter and R. Silbey, Phys. Rev. Lett. 44 (1980) 55.

[14] M. Lax and T. Odagaki, in: Macroscopic properties of disordered media, ed. R. Burridge (Springer, Berlin, 1982) p. 148.

[15] T. Odagaki and M. Lax, Phys. Rev. B24 (1981) 5284

[16] A. Miller and E. Abrahams, Phys. Rev. 120 (1960) 745.

[17] J. Bernasconi, H.U. Beyeler, S. Strässler and S. Alexander, Phys. Rev. Lett. 42 (1979) 819.

[18] H. Scher and E.W. Montroll, Phys. Rev. B1 2 (1975) 2455.

[19] H. Namikawa, J. Non-Cryst. Solids 18 (1975) 173.

[20] M. Abkowitz, P.G. Le Comber and W.E. Spear, Comm. Phys. 1 (1976) 175.

[21] M.S. Frost and A.K. Jonscher, Thin Solid Films 29 (1975) 7.

[22] A.R. Long and N. Balkan, J. Non-Cryst. Solids 35-36 (1980) 415.

[23] M. Suzuki, J. Phys. Chem. Solids 41 (1980) 1253.

[24] M. Careem and A.K. Jonscher, Philos. Mag. 35 (1977) 1489.

[25] S.R. Elliott, Philos. Mag. B40 (1979) 507. 